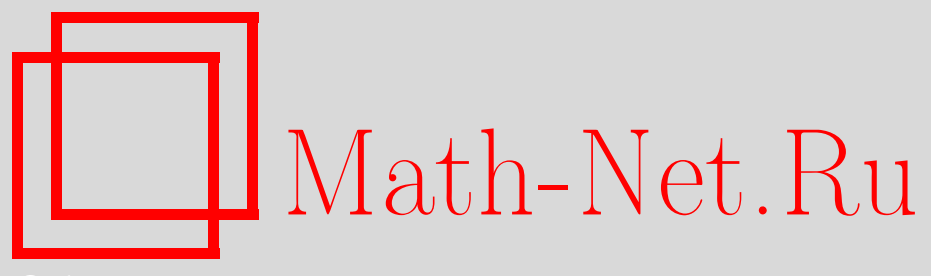

Л. М. Арутюнян, Е. Д. Косов, Оценки интегральных норм многочленов на пространствах с выпуклыми мерами, Матем. сб., 2015, том 206, номер 8, 3-22

DOI: https://doi.org/10.4213/sm8436

Использование Общероссийского математического портала Math-Net.Ru подразумевает, что вы прочитали и согласны с пользовательским соглашением http://www . mathnet.ru/rus/agreement

Параметры загрузки:

IP : 54.84 .234 .179

26 апреля 2023 г., 12:53:51

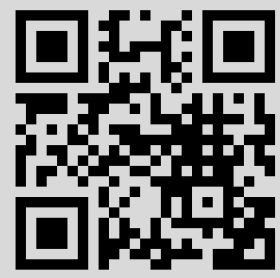




\author{
Л. М. Арутюнян, Е. Д. Косов
}

\title{
Оценки интегральных норм многочленов на пространствах с выпуклыми мерами
}

\begin{abstract}
В работе доказано, что измеримые многочлены степени $d$ интегрируемы по выпуклой мере в любой положительной степени, а все их $L^{p}$-нормы эквивалентны. Также доказывается закон нуля или единицы для множеств уровня измеримых многочленов и множеств сходимости измеримых многочленов на пространствах с выпуклыми мерами. Для непрерывных многочленов получена оценка $L^{1}$-нормы через $L^{1}$-норму их сужений на какое-либо множество положительной меры.

Библиография: 19 названий.
\end{abstract}

Ключевые слова: выпуклые меры, логарифмически вогнутые меры, измеримые многочлены.

DOI: $10.4213 / \mathrm{sm} 8436$

\section{Введение}

Хорошо известно, что измеримые многочлены на бесконечномерных пространствах с гауссовскими мерами обладают целым рядом полезных свойств (см. [1], [2]), к важнейшим из которых можно отнести следующие:

1) на пространстве многочленов фиксированной степени эквивалентны все $L^{p}$-нормы;

2) из сходимости по мере последовательности многочленов фиксированной степени на множестве положительной меры следует их сходимость во всех $L^{p}$ на всем пространстве;

3) для множеств уровня многочленов фиксированной степени и множеств их сходимости справедлив закон нуля или единицы, т.е. такие множества могут иметь меру только 0 или 1.

Некоторые из подобных свойств уже были перенесены на случай выпуклых мер. Так, в [3] доказано, что измеримые полунормы интегрируемы по выпуклой мере в любой степени. Из этого можно вывести, что всякий непрерывный многочлен лежит в $L^{1}$ по всякой выпуклой мере, а также, что все $L^{p}$-нормы на пространстве всех измеримых линейных функций, т.е. многочленов степени 1 , эквивалентны. Однако не было известно, всякий ли измеримый многочлен интегрируем по выпуклой мере. В нашей работе дается положительный ответ

Исследование выполнено за счет гранта Российского научного фонда (проект № 14-11-00196). 
на этот вопрос. Более того, ниже показано, что измеримые многочлены интегрируемы в любой положительной степени, а все их $L^{p}$-нормы эквивалентны, причем для измеримых многочленов $f$ степени $d$ верна оценка

$$
\|f\|_{p} \leqslant C(p, d)\|f\|_{0}
$$

где $C(p, d)=\left(c_{1} p d\right)^{d}$, а $c_{1}-$ универсальная постоянная. В конечномерном случае этот результат был получен С. Г. Бобковым (см. [4]), а затем распространен В. Е. Бережным (см. [5]) на замыкание конечномерных многочленов, однако остается неизвестным, приближаются ли измеримые многочлены конечномерными. Отметим, что подобные вопросы также изучались, например, в работах [6], [7].

В работе также доказывается закон нуля или единицы для множеств уровня измеримых многочленов и множеств сходимости измеримых многочленов фиксированной степени на пространствах с выпуклыми мерами.

Наконец, для непрерывных многочленов нами получена оценка $L^{1}$-нормы через $L^{1}$-норму их сужений на какое-либо множество положительной меры. Из этого, в частности, следует эквивалентность этих норм на пространстве многочленов фиксированной степени. Для счетных произведений выпуклых мер с конечномерными сомножителями эквивалентность этих норм также была установлена В. Е. Бережным.

Напомним некоторые понятия и факты. Пусть $\mu$ - вероятностная радоновская мера на локально выпуклом пространстве $E$ с топологическим сопряженным $E^{*}$ (см. [8]). Положим

$$
\|f\|_{p}=\left(\int_{E}|f|^{p} d \mu\right)^{1 / p}, \quad p>0, \quad\|f\|_{0}=\exp \left(\int_{E} \ln |f| d \mu\right)=\lim _{r \rightarrow 0}\|f\|_{r} .
$$

Выпуклой (или логарифмически вогнутой) называют такую вероятностную радоновскую меру $\mu$ на $E$, что всякий ее конечномерный образ (т.е. образ при всяком непрерывном конечномерном линейном отображении) является выпуклой мерой на $\mathbb{R}^{n}$, т.е. задается плотностью вида $e^{-V}$ относительно меры Лебега на некотором аффинном подпространстве, где $V$ - выпуклая функция (возможно, с бесконечными значениями) на этом подпространстве (см. [9]).

Мы будем использовать и другое эквивалентное определение выпуклой меры, состоящее в выполнении для всяких борелевских множеств $A, B$ неравенства

$$
\mu_{*}(\lambda A+(1-\lambda) B) \geqslant \mu(A)^{\lambda} \mu(B)^{1-\lambda},
$$

где $\lambda \in[0,1]-$ произвольное число и $\mu_{*}-$ внутренняя мера (см. [3]).

Назовем мономом степени $m$ функцию вида $B_{m}(x, \ldots, x): E \rightarrow \mathbb{R}$, где $B\left(x_{1}\right.$, $\left.\ldots, x_{m}\right)-m$-линейная симметричная функция на $E^{m}$. Алгебраическим многочленом степени $d$ будем называть функцию $f$ вида

$$
f(x)=\sum_{m=0}^{d} B_{m}(x, \ldots, x),
$$

где $B_{d}$ не является тождественно нулевым. 
Через $\mathscr{P}_{\text {mes }}^{d}(\mu)$ будем обозначать множество алгебраических многочленов степени $d$, которые являются измеримыми относительно меры $\mu$. Будем называть функции из $\mathscr{P}_{\text {mes }}^{d}(\mu)$ измеримыми многочленами степени $d$.

Обозначим через $\mathscr{P}_{\text {cont }}^{d}(\mu)$ совокупность таких измеримых многочленов, которые лежат в замыкании множества непрерывных алгебраических многочленов степени $d$ в $L^{1}(\mu)$ (это множество совпадает с пределами по мере таких многочленов, что легко видеть, например, из следствия 1.2). Отметим, что если функция является пределом по мере непрерывных многочленов, то у нее есть версия из $\mathscr{P}_{\text {cont }}^{d}(\mu)$ (см. [10]).

Неизвестно, совпадают ли классы $\mathscr{P}_{\text {mes }}^{d}(\mu)$ и $\mathscr{P}_{\text {cont }}^{d}(\mu)$, как это имеет место для гауссовской меры.

Приведем пример измеримого линейного функционала, который не является непрерывным. Рассмотрим в качестве пространства $E$ пространство $\mathbb{R}^{\infty}$ с выпуклой мерой $\mu$. Положим

$$
\ell(x)=\sum_{i=1}^{\infty} c_{i} x_{i}
$$

где $c_{i}=2^{-i}\left\|x_{i}\right\|_{L^{1}(\mu)}^{-1}$, на линейном подпространстве сходимости данного ряда и продолжим на все пространство до линейной функции. Функционал $\ell(x)$ не является непрерывным, поскольку зависит от бесконечного числа переменных. Другие примеры измеримых многочленов можно найти в [1], [10], [11], где рассматриваются многочлены, измеримые по гауссовской мере.

\section{§ 1. Интегрируемость измеримых многочленов и связанные с этим оценки}

Пусть $\Delta-$ некоторый отрезок в локально выпуклом пространстве $E$, т.е. выпуклая оболочка двух точек. Обозначим через $m_{\Delta}$ нормированную меру Лебега на отрезке $\Delta$, т.е. такую меру, что

$$
m_{\Delta}(A)=\frac{m(A \cap \Delta)}{m(\Delta)},
$$

где $m$ - стандартная мера Лебега (возникающая путем выбора масштаба на соответствующей прямой; выбор масштаба в дальнейшем не играет роли, поскольку мы используем нормированную меру $\left.m_{\Delta}\right)$.

Пусть $A$ - борелевское множество в $E$. Для всякого $\lambda \geqslant 1$ определим множество $A_{\lambda}$ следующим образом:

$$
A_{\lambda}=\left\{x \in A: m_{\Delta}(A) \geqslant 1-\frac{1}{\lambda} \text { для всякого интервала } \Delta \ni x\right\} .
$$

В работе [12] доказан следующий бесконечномерный аналог так называемой геометрической КЛШ-леммы (геометрической леммы Каннана-Ловаса-Шимоновича) из [13]. 
Теорема. Пусть $\mu$ - выпуклая радоновская мера на полном локально въпуклом пространстве Е. Тогда для всякого борелевского множества $A$ и для всякого $\lambda \geqslant 1$ имеем

$$
\mu^{*}\left(A_{\lambda}\right) \leqslant(\mu(A))^{\lambda}
$$

где $\mu^{*}$ - внешняя мера.

Напомним также неравенство Ремеза (см. [14], [15]).

Неравенство Ремеза. Пусть $f$ - многочлен степени $d$ на вещественной прямой. Тогда для всякого интервала $\Delta$ и всякого измеримого множества $J \subset \Delta$ положительной меры выполнена оценка

$$
\max _{\Delta}|f| \leqslant\left(\frac{4}{m_{\Delta}(J)}\right)^{d} \sup _{J}|f| .
$$

Наш первый результат - закон нуля или единицы.

Теорема 1.1. Пусть $\mu$-выпуклая радоновская мера на локалъно выпуклом пространстве $E, f$ - измеримый многочлен степени $d u\left\{f_{n}\right\}$ - последовательность многочленов степени $d$. Тогда для всякой постоянной $C$ число $\mu(\{x$ : $f(x)=C\})$ равно либо 0, либо 1. Кроме того, число $\mu\left(\left\{x: \exists \lim _{n \rightarrow \infty} f_{n}(x)\right\}\right)$ также равно либо 0 , либо 1.

ДокАЗАтельство. Заметим, что достаточно доказать это утверждение только для полных пространств. Действительно, предположим, что оно верно для полных пространств, но $E$ неполно. Обозначим через $\bar{E}$ пополнение пространства $E$, т.е. $\bar{E}$ - полное локально выпуклое пространство, в котором $E$ является всюду плотным линейным подпространством. Пусть $F$ - алгебраическое дополнение к $E$ в пространстве $\bar{E}$, т.е. всякий элемент $x \in \bar{E}$ можно единственным образом представить в виде суммы $x=y+z$, где $y \in E, z \in F$. Продолжим многочлен $f$ на пространство $\bar{E}$ следующим образом: $f(x)=f(y+z):=f(y)$. Меру тоже можно продолжить естественным образом по формуле $\mu(\bar{E} \backslash E)=0$. Таким образом, по предположению утверждение верно для многочлена $f$ на $\bar{E}$, а так как $\mu(E)=1$, то и для многочлена $f$ на $E$.

Теперь считаем $E$ полным пространством. Сначала докажем первое утверждение. Ясно, что достаточно доказать его для множеств $\{x: f(x)=0\}$. Положим $A=\{x:|f(x)|>0\}$. Пусть $A^{\prime}$ - такое борелевское множество, что $A \subset A^{\prime}$ и $\mu\left(A^{\prime}\right)=\mu(A)$. Заметим, что $A \subset A_{\lambda}^{\prime}$. Действительно, пусть $x \in A$. Тогда ограничение $f$ на всякую прямую, проходящую через $x$, оказывается ненулевым многочленом, значит, $m_{\Delta}(A)=1$, поэтому

$$
m_{\Delta}\left(A^{\prime}\right) \geqslant m_{\Delta}(A)=1>1-\frac{1}{\lambda} .
$$

Применяя бесконечномерную геометрическую КЛШ-лемму, имеем

$$
\mu(A) \leqslant \mu^{*}\left(\left(A^{\prime}\right)_{\lambda}\right) \leqslant\left(\mu\left(A^{\prime}\right)\right)^{\lambda}=(\mu(A))^{\lambda} .
$$

Таким образом, $\mu(A) \leqslant(\mu(A))^{\lambda}$, откуда сразу следует первое утверждение теоремы. 
Для доказательства второго утверждения достаточно рассмотреть множество

$$
A=\left\{x: \nexists \lim _{n \rightarrow \infty} f_{n}(x)\right\}
$$

и провести аналогичные рассуждения. Для доказательства того, что $A \subset A_{\lambda}^{\prime}$, воспользуемся тем, что последовательность многочленов степени $d$ на прямой, которая не сходится в некоторой точке, не может сходиться на множестве положительной меры.

ЗАмечание 1.1. Для сфер, т.е. множеств вида $g^{-1}(\{1\})$, где $g$ - полунорма, закон нуля или единицы неверен даже для гауссовских мер. Достаточно рассмотреть меру $\gamma$ на $\mathbb{R}^{\infty}$, которая является счетной степенью стандартной гауссовской меры на прямой, и полунорму

$$
g(x)=\max \left(\left|x_{1}\right|, \sqrt{B(x, x)}\right),
$$

где $B(x, x)$ - это продолжение на все пространство полунормы

$$
\limsup _{n \rightarrow \infty} \frac{1}{n} \sum_{i=1}^{n} x_{i}^{2}
$$

с линейного пространства, на котором указанный верхний предел конечен.

Про закон нуля или единицы см. также замечание 2.1 и лемму 2.4.

Доказательство следующей теоремы - несложная модификация доказательства леммы о сравнении из [13].

Теорема 1.2. Пусть $\mu$ - выпуклая радоновская мера на локально выпуклом пространстве $E, f$ - измеримый многочлен степени d. Тогда верна следующая оценка:

$$
\mu\left(\left\{|f| \geqslant(4 \lambda)^{d} k\right\}\right) \leqslant(\mu(\{|f| \geqslant k\}))^{\lambda} .
$$

ДокАЗАтЕльство. Аналогично теореме 1.1 случай неполного пространства $E$ сводится к случаю полного пространства, поэтому считаем пространство $E$ полным. Если $f$ совпадает с постоянной $\mu$-п.в., то утверждение очевидно. Пусть теперь это не так, тогда мера всякой линии уровня $f$ нулевая по теореме 1.1. Пусть

$$
A=\{|f| \geqslant k\}
$$

и $A^{\prime}$ - такое борелевское множество, что $A \subset A^{\prime}$ и $\mu(A)=\mu\left(A^{\prime}\right)$; такое множество существует ввиду измеримости $A$. Пусть $x \in A^{\prime}$ и $x \notin\left(A^{\prime}\right)_{\lambda}$. Тогда найдется такой интервал $\Delta$, что $x \in \Delta$ и $m_{\Delta}\left(\Delta \backslash A^{\prime}\right)>\frac{1}{\lambda}$. Воспользуемся неравенством Ремеза:

$$
|f(x)| \leqslant \max _{\Delta}|f| \leqslant\left(\frac{4}{m_{\Delta}\left(\Delta \backslash A^{\prime}\right)}\right)^{d} \sup _{\Delta \backslash A^{\prime}}|f|<(4 \lambda)^{d} k .
$$

Таким образом, $\left\{x \in A^{\prime}:|f(x)| \geqslant(4 \lambda)^{d} k\right\} \subset\left(A^{\prime}\right)_{\lambda}$ и

$$
\mu\left(\left\{x \in A^{\prime}:|f(x)| \geqslant(4 \lambda)^{d} k\right\}\right) \leqslant \mu^{*}\left(\left(A^{\prime}\right)_{\lambda}\right) \leqslant\left(\mu\left(A^{\prime}\right)\right)^{\lambda}=(\mu(A))^{\lambda} .
$$


Далее,

$$
\begin{gathered}
\mu\left(\left\{|f| \geqslant(4 \lambda)^{d} k\right\}\right)=\mu\left(\left\{x \in A:|f(x)| \geqslant(4 \lambda)^{d} k\right\}\right) \\
=\mu\left(\left\{x \in A^{\prime}:|f(x)| \geqslant(4 \lambda)^{d} k\right\}\right),
\end{gathered}
$$

поэтому

$$
\mu\left(\left\{|f| \geqslant(4 \lambda)^{d} k\right\}\right) \leqslant(\mu(A))^{\lambda},
$$

что и дает требуемое.

СлеДСТвИЕ 1.1. Пусть $\mu$ - выпуклая радоновская мера на локалъно выпуклом пространстве $E, f$ - измеримый многочлен степени $d$, не равный постоянной $\mu$-п.в. Тогда найдется такое положительное число $k(f)$, что для всякого $\lambda>1$ выполнены оценки

$$
\mu\left(\left\{x:|f(x)| \geqslant(4 \lambda)^{d} k(f)\right\}\right) \leqslant e^{-\lambda}, \quad \mu\left(\left\{x:|f(x)| \leqslant(4 \lambda)^{-d} k(f)\right\}\right) \leqslant \frac{1}{\lambda} .
$$

ДокАЗАТЕЛЬСтво. Поскольку $f$ не совпадает с постоянной $\mu$-п.в., по теореме 1.1 мера всякой линии уровня $f$ нулевая. Пусть

$$
k(f)=\inf \left\{k: \mu(\{|f| \geqslant k\}) \leqslant \frac{1}{e}\right\}>0,
$$

тогда $\mu(\{|f| \geqslant k(f)\})=\frac{1}{e}$. Поэтому первая оценка - прямое следствие теоремы 1.2 для $k=k(f)$. Докажем теперь вторую оценку. Применим теорему 1.2 для $k=(4 \lambda)^{-d} k(f)$ :

$$
\frac{1}{e} \leqslant\left(1-\mu\left(\left\{x:|f(x)|<(4 \lambda)^{-d} k(f)\right\}\right)\right)^{\lambda} .
$$

Так как мера всякой линии уровня $f$ нулевая, то

$$
\mu\left(\left\{x:|f(x)| \leqslant(4 \lambda)^{-d} k(f)\right\}\right)=\mu\left(\left\{x:|f(x)|<(4 \lambda)^{-d} k(f)\right\}\right) \leqslant 1-e^{-1 / \lambda} \leqslant \frac{1}{\lambda},
$$

что завершает доказательство.

ТЕОРема 1.3. Пусть $\mu$ - выпуклая радоновская мера на локально выпуклом пространстве $E, f$ - измеримый многочлен степени $d$. Тогда $f \in L^{p}(\mu)$ nри всех $p>0$. Кроме того, если $f$ не совпадает с константой п.в., то верна оценка

$$
\|f\|_{p} \leqslant k(f) 4^{d}(1+p d \Gamma(p d))^{1 / p},
$$

где $k(f)$ - число из предыдущего следствия. При $p d \geqslant 1$ выполняется также оченка

$$
\|f\|_{p} \leqslant k(f)\left(c_{1} p d\right)^{d}
$$

где $c_{1}>1$ - абсолютная константа, не зависящая от $d u p$. 
ДокАЗАтЕльство. Если $f$ совпадает с постоянной $\mu$-п.в., то утверждение очевидно. Пусть теперь $f$ не совпадает с постоянной $\mu$-п.в. Тогда

$$
\int_{E}|f|^{p} d \mu=\int_{0}^{\infty} \mu\left(\left\{|f|^{p} \geqslant t\right\}\right) d t=p \int_{0}^{\infty} t^{p-1} \mu(\{|f| \geqslant t\}) d t .
$$

Производя в последнем интеграле замену $t=(4 \lambda)^{d} k(f)$, получаем

$$
\begin{aligned}
& k(f)^{p} 4^{p d} p d \int_{0}^{\infty} \lambda^{d(p-1)+d-1} \mu\left(\left\{|f| \geqslant(4 \lambda)^{d} k(f)\right\}\right) d \lambda \\
& =k(f)^{p} 4^{p d} p d\left(\int_{0}^{1}+\int_{1}^{\infty}\right) \lambda^{p d-1} \mu\left(\left\{|f| \geqslant(4 \lambda)^{d} k(f)\right\}\right) d \lambda .
\end{aligned}
$$

Теперь меру множества в первом интеграле оценим единицей, а во втором интеграле - по следствию 1.1. Тем самым последнее выражение оценится через

$$
k(f)^{p} 4^{p d}+k(f)^{p} 4^{p d} p d \int_{1}^{\infty} \lambda^{p d-1} e^{-\lambda} d \lambda \leqslant k(f)^{p} 4^{p d}(1+p d \Gamma(p d))<\infty .
$$

Оценка при $p d \geqslant 1$ следует из уже полученной и того, что $\Gamma(t) \leqslant t^{t}$ при $t \geqslant 1$, что дает первую оценку из условий теоремы.

Отметим, что, так как

$$
k(f) \leqslant e \int|f| d \mu,
$$

то в предыдущей теореме доказана эквивалентность $L^{p}$ и $L^{1}$-норм на пространстве измеримых многочленов фиксированной степени. В конечномерном случае доказана более точная оценка $\|f\|_{p} \leqslant(c p)^{d}\|f\|_{1}$, где $f$ - многочлен степени $d$ и $c$ - некоторая универсальная постоянная (см. [16]).

Следующая теорема обобщает другой результат С. Г. Бобкова (см. [4]) на случай измеримых многочленов.

ТЕОРема 1.4. Пусть $\mu$ - выпуклая радоновская мера на локалъно выпуклом пространстве $E, f$ - измеримый многочлен степени $d$. Тогда при $q>0$ имеем

$$
\|f\|_{q} \leqslant c(q, d)\|f\|_{0}
$$

где $c(q, d)$ - постоянная, зависящая только от $q, d$. При $q d \geqslant 1$ в качестве $c(q, d)$ можно взять $\left(c_{2} q d\right)^{d}$, где $c_{2}-$ абсолютная константа, не зависящая om $q, d$.

ДокАЗАтЕЛЬСтво. Если $f$ совпадает с постоянной $\mu$-П.в., то утверждение очевидно. Пусть теперь $f$ не совпадает с постоянной $\mu$-п.в. Мы получим оценку $\|f\|_{0}$ снизу через $k(f)$. Воспользуемся тем, что

$$
\|f\|_{0}=\lim _{p \rightarrow 0}\|f\|_{p}
$$

и оценим сначала $\|f\|_{p}$ :

$$
\int_{E}|f|^{p} d \mu=\int_{0}^{\infty} \mu\left(\left\{|f|^{p} \geqslant t\right\}\right) d t=p \int_{0}^{\infty} t^{p-1} \mu(\{|f| \geqslant t\}) d t .
$$


В последнем интеграле производим замену $t=(4 \lambda)^{-d} k(f)$ :

$$
\begin{aligned}
& k(f)^{p} c^{-p d} p d \int_{0}^{\infty} \lambda^{-p d-1} \mu\left(\left\{|f| \geqslant(4 \lambda)^{-d} k(f)\right\}\right) d \lambda \\
& \geqslant k(f)^{p} 4^{-p d} p d \int_{1}^{\infty} \lambda^{-p d-1} \mu\left(\left\{|f| \geqslant(4 \lambda)^{-d} k(f)\right\}\right) d \lambda \\
& =k(f)^{p} c^{-p d}-k(f)^{p} c^{-p d} p d \int_{1}^{\infty} \lambda^{-p d-1} \mu\left(\left\{|f|<(4 \lambda)^{-d} k(f)\right\}\right) d \lambda .
\end{aligned}
$$

Пользуясь следствием 1.1, оценим последнее выражение через

$$
k(f)^{p} 4^{-p d}-k(f)^{p} 4^{-p d} p d \int_{1}^{\infty} \lambda^{-2-p d} d \lambda=k(f)^{p} 4^{-p d}\left(1-\frac{p d}{p d+1}\right)=\frac{k(f)^{p} 4^{-p d}}{p d+1} .
$$

Таким образом,

$$
\|f\|_{p} \geqslant \frac{k(f) 4^{-d}}{(p d+1)^{1 / p}}
$$

Теперь, устремляя $p$ к нулю, получаем, что $\|f\|_{0} \geqslant k(f)(4 e)^{-d}$. По теореме 1.3 имеем $\|f\|_{q} \leqslant k(f) 4^{d}(1+q d \Gamma(q d))^{1 / q}$. Поэтому

$$
\|f\|_{q} \leqslant(16 e)^{d}(1+q d \Gamma(q d))^{1 / q}\|f\|_{0} .
$$

Оценка при $q d \geqslant 1$ следует из последнего утверждения теоремы 1.3.

Приведем в качестве следствия вышеизложенного обобщение неравенства из статьи [17] на случай измеримых многочленов.

СлЕДСТвиЕ 1.2. Пусть $\mu$ - выпуклая радоновская мера на локально выпуклом пространстве $E, f$ - измеримый многочлен степени $d$. Тогда при $p d \geqslant 1$ имеет место неравенство

$$
\mu(\{|f| \leqslant t\})\|f\|_{p}^{1 / d} \leqslant c_{1} t^{1 / d} p d,
$$

где $c_{1}$ - абсолютная постоянная, не зависящая от $p$ ud.

ДокАЗАтЕльство. Если $f$ совпадает с постоянной $\mu$-п.в., то утверждение очевидно. Пусть теперь $f$ не совпадает с постоянной $\mu$-п.в. Подставив $\lambda=$ $\left(4 t^{-1} k(f)\right)^{1 / d}$ в неравенство

$$
\mu\left(\left\{x:|f(x)| \leqslant(4 \lambda)^{-d} k(f)\right\}\right) \leqslant \frac{1}{\lambda}
$$

из следствия 1.1 , получим

$$
\mu(\{|f| \leqslant t\}) \leqslant \frac{t^{1 / d}}{4^{1 / d}}(k(f))^{-1 / d} .
$$

Воспользуемся оценкой

$$
(k(f))^{-1 / d} \leqslant\|f\|_{p}^{-1 / d}\left(c_{1} p d\right),
$$


которая следует из неравенства в теореме 1.3, и получим неравенство

$$
\mu(\{|f| \leqslant t\})\|f\|_{p}^{1 / d} \leqslant \frac{t^{1 / d}}{4^{1 / d}} c_{1} p d .
$$

Для завершения доказательства достаточно заметить, что $4^{1 / d} \geqslant 1$.

ЗАмЕЧАнИЕ 1.2 . Пусть $F$ - измеримое полиномиальное отображение со значениями в банаховом пространстве $(X,\|\cdot\|)$ с сопряженным $\left(X^{*},\|\cdot\|^{*}\right)$. Положим $f(x)=\|F(x)\|$. Тогда для ограничения $f$ на любую прямую в пространстве $X$ выполняется неравенство Ремеза, поэтому нетрудно доказать аналоги вышеизложенных утверждений для таких функций $F$. Оценка Ремеза в этом случае следует из того, что если $\Delta$ - отрезок в $E$ и $J$ - борелевское подмножество $\Delta$, то

$$
\sup _{J}\|F(x)\|=\sup _{J} \sup _{\|\ell\|^{*}=1} \ell(F(x))
$$

a $\ell(F(\cdot))$ - уже вещественный многочлен на пространстве $E$, к которому можно применить обычное неравенство Ремеза.

\section{§ 2. Оценка интегральной нормы непрерывного многочлена через интегральную норму его сужения на подмножество}

Основной результат этого параграфа заключается в следующей теореме.

ТеОРема 2.1. Пусть $\mu$-выпуклая радоновская мера на локалъно выпуклом пространстве Е и $U$ - измеримое множество положительной меры. Пусть $f \in \mathscr{P}_{\text {cont }}^{d}(\mu)$. Тогда

$$
\mu(U)^{d+1} \int_{E}|f| d \mu \leqslant(C d)^{2 d} \int_{U}|f| d \mu,
$$

где $C$ - некоторая абсолютная постоянная. Другими словами,

$$
\|f\|_{L^{1}(\mu)} \leqslant \frac{(C d)^{2 d}}{\mu(U)^{d}}\|f\|_{L^{1}\left(\mu_{U}\right)}, \quad \text { əде } \quad \mu_{U}(A)=\frac{\mu(A \cap U)}{\mu(U)} .
$$

Отметим, что в [17] подобная оценка доказана для мер, которые являются равномерными распределениями на выпуклых телах в конечномерном пространстве. При этом использовалась оценка $L^{\infty}$-нормы многочлена через его $L^{1}$-норму, которая зависит от размерности. Поэтому случай произвольной выпуклой меры не сводится к данному.

Напомним, что функция называется полунепрерывной снизу, если

$$
\liminf _{x \rightarrow x_{0}} f(x) \geqslant f\left(x_{0}\right) .
$$

Функция называется полунепрерывной сверху, если $\limsup _{x \rightarrow x_{0}} f(x) \leqslant f\left(x_{0}\right)$. Таким образом, индикатор открытого множества - полунепрерывная снизу функция, а индикатор замкнутого множества - полунепрерывная сверху.

В доказательстве используется следующий результат из [12], обобщающий локализационную лемму из [18] на бесконечномерное пространство. 
Теорема. Пусть $\mu$ - выпуклая радоновская мера на полном локально въпуклом пространстве $E$. Пусть $u, v: E \rightarrow \mathbb{R}$ - такие полунепрерывные снизу н-интегрируемые функиии, что

$$
\int_{E} u d \mu>0, \quad \int_{E} v d \mu>0 .
$$

Тогда найдутся точки $a, b \in E$ и выпуклая мера $\nu$ на отрезке $\Delta=[a, b]$ maкue, чmo

$$
\int_{\Delta} u d \nu>0, \quad \int_{\Delta} v d \nu>0 .
$$

Следующее утверждение - перенос другой известной локализационной леммы (см. [19]) на бесконечномерное пространство.

СледСтвиЕ 2.1. Пусть $f_{1}, f_{2}$ - две полунепрерывные снизу неотрицательнъе функиии на $E, f_{3}, f_{4}$ - две полунепрерывные сверху неотрицательные функиии на Е. Предположим, что $f_{1}^{\alpha} f_{2}^{\beta} \leqslant f_{3}^{\alpha} f_{4}^{\beta}$ и для всякого отрезка $\Delta=$ $[a, b] \subset E$ и всякой меры $\nu$ слотностъю $\exp \ell$ относительно меры Лебега на отрезке $\Delta$, где $\ell$ - аффинная функиия на $\Delta$, выполнено неравенство

$$
\left(\int_{\Delta} f_{1} d \nu\right)^{\alpha}\left(\int_{\Delta} f_{2} d \nu\right)^{\beta} \leqslant\left(\int_{\Delta} f_{3} d \nu\right)^{\alpha}\left(\int_{\Delta} f_{4} d \nu\right)^{\beta} .
$$

Тогда для всякой выпуклой радоновской меры $\mu$ на Е выполнено неравенство

$$
\left(\int_{E} f_{1} d \mu\right)^{\alpha}\left(\int_{E} f_{2} d \mu\right)^{\beta} \leqslant\left(\int_{E} f_{3} d \mu\right)^{\alpha}\left(\int_{E} f_{4} d \mu\right)^{\beta} .
$$

ДокАзАТЕльство. Будем пользоваться тем, что это утверждение верно в конечномерном случае (см. [19]), но при этом будем использовать также фрагменты доказательства из цитированной работы. Предположим, что для некоторой вероятностной радоновской выпуклой меры $\mu$ выполнено обратное строгое неравенство. Тогда

$$
\int_{E} f_{2}>0
$$

Добавлением константы можно добиться того, что

$$
\int_{E} f_{3}>0
$$

Значит, найдется число $A$ такое, что

$$
\int_{E} f_{1} d \mu\left(\int_{E} f_{3} d \mu\right)^{-1}>A^{1 / \alpha}, \quad \int_{E} f_{4} d \mu\left(\int_{E} f_{2} d \mu\right)^{-1}<A^{1 / \beta} .
$$

Тогда

$$
\int_{E} f_{1}-A^{1 / \alpha} f_{3} d \mu>0, \quad \int_{E} A^{1 / \beta} f_{2}-f_{4} d \mu>0
$$


причем $f_{1}-A^{1 / \alpha} f_{3}$ и $A^{1 / \beta} f_{2}-f_{4}-$ полунепрерывные снизу функции, поэтому найдутся отрезок $\Delta$ и вероятностная выпуклая мера $\nu$ на нем, для которых

$$
\int_{\Delta} f_{1}-A^{1 / \alpha} f_{3} d \nu>0, \quad \int_{\Delta} A^{1 / \beta} f_{2}-f_{4} d \nu>0 .
$$

Следовательно,

$$
\left(\int_{\Delta} f_{1} d \nu\right)^{\alpha}\left(\int_{\Delta} f_{2} d \nu\right)^{\beta}>\left(\int_{\Delta} f_{3} d \nu\right)^{\alpha}\left(\int_{\Delta} f_{4} d \nu\right)^{\beta},
$$

что противоречит конечномерной локализационной лемме.

Определим при $r \leqslant s$ функции

$$
P(r, s)=\int_{r}^{s}|t| e^{t} d t, \quad Q(r, s)=\int_{r}^{s} e^{t} d t, \quad F(r, s)=\frac{P(r, s)}{(Q(r, s))^{2}},
$$

причем $F(r, r)$ определяется по непрерывности, в частности, $F(0,0)=\frac{1}{2}$.

ЛЕмма 2.1. Функиия $F(r, s)$ обладает следующими свойствами:

1) убывает по $s$, если $s \geqslant r \geqslant 0$ или $r \leqslant s \leqslant 0$,

2) возрастает по $r$, если $r \geqslant 0$,

3) убъвает по $r$, если $s=0, r \leqslant 0$.

ДокАзАТЕЛЬство. Найдем производную $F$ по $s$ :

$$
\partial_{s} F=\left(\int_{r}^{s} e^{t} d t\right)^{-3}\left(e^{s}|s| \int_{r}^{s} e^{t} d t-2 e^{s} \int_{r}^{s}|t| e^{t} d t\right) .
$$

Положим

$$
\varphi(s)=|s| \int_{r}^{s} e^{t} d t-2 \int_{r}^{s}|t| e^{t} d t .
$$

Имеем $\varphi(r)=0$, а также $\varphi^{\prime}(s)=\operatorname{sgn}(s)\left(e^{s}-e^{r}\right)-|s| e^{s}$. При $r \geqslant 0$ верна оценка

$$
\varphi^{\prime}(s)=e^{s}-e^{r}-s e^{s}=1-e^{r}-e^{s}\left(e^{-s}-(1-s)\right) \leqslant 0 .
$$

При $r \leqslant s \leqslant 0$ верна оценка

$$
\varphi^{\prime}(s)=-e^{s}+e^{r}+s e^{s} \leqslant 0 .
$$

Поэтому $\varphi(s) \leqslant 0$, и значит, $\partial_{s} F \leqslant 0$, что доказывает п. 1$)$.

Найдем теперь производную $F$ по $r$ :

$$
\partial_{r} F=\left(\int_{r}^{s} e^{t} d t\right)^{-3}\left(-e^{r}|r| \int_{r}^{s} e^{t} d t+2 e^{r} \int_{r}^{s}|t| e^{t} d t\right) .
$$

Положим

$$
\psi(r)=-|r| \int_{r}^{s} e^{t} d t+2 \int_{r}^{s}|t| e^{t} d t
$$

Тогда $\psi^{\prime}(r)=-\operatorname{sgn}(r)\left(e^{s}-e^{r}\right)-|r| e^{r}$. В случае $r \geqslant 0$ имеем $\psi^{\prime}(r) \leqslant 0, \psi(s)=0$, поэтому $\psi(r) \geqslant 0$, что доказывает п. 2).

В случае $r \leqslant 0, s=0$ имеем $\psi^{\prime}(r)=e^{s}-1+e^{r}\left(r-1+e^{-r}\right) \geqslant 0, \psi(0)=0$, что доказывает п. 3). Лемма доказана. 
СлЕДСТВИЕ 2.2. При $r \leqslant 0 u s \geqslant 0$ верны неравенства

$$
\frac{1}{2}=F(0,0) \leqslant F(r, 0) \leqslant F(-\infty, 0)=1, \quad F(0, s) \leqslant F(0,0)=\frac{1}{2}<1 .
$$

Лемма 2.2. Пусть $r \leqslant 0 \leqslant s$. Тогда

$$
4^{-1} F(0, s) \leqslant F(r, s) \leqslant 4 F(0, s), \quad F(r, s) \leqslant 4 F(r, 0) .
$$

ДокАзАтельство. Докажем сначала оценку $F(r, s) \leqslant 4 F(0, s)$. Легко видеть, что

$$
F(r, s)=\frac{P(r, 0)+P(0, s)}{(Q(r, 0)+Q(0, s))^{2}} \leqslant \frac{P(r, 0)+P(0, s)}{Q(r, 0)^{2}+Q(0, s)^{2}} .
$$

Поскольку $F(r, 0) \leqslant 1$ по следствию 2.2 , то

$$
\frac{P(r, 0)+P(0, s)}{Q(r, 0)^{2}+Q(0, s)^{2}} \leqslant \frac{Q(r, 0)^{2}+P(0, s)}{Q(r, 0)^{2}+Q(0, s)^{2}} .
$$

Так как функция $\frac{t+a}{t+b}, 0<a<b$, возрастает при $t>0$ и $F(0, s)<1$ в силу следствия 2.2, то полученное выше выражение оценивается как

$$
\frac{Q(-\infty, 0)^{2}+P(0, s)}{Q(-\infty, 0)^{2}+Q(0, s)^{2}}=\left(1+\int_{0}^{s}|t| e^{t} d t\right)\left(1+\left(\int_{0}^{s} e^{t} d t\right)^{2}\right)^{-1} .
$$

Покажем, что

$$
\left(1+\int_{0}^{s}|t| e^{t} d t\right)\left(1+\left(\int_{0}^{s} e^{t} d t\right)^{2}\right)^{-1} \leqslant 4 \int_{0}^{s}|t| e^{t} d t\left(\int_{0}^{s} e^{t} d t\right)^{-2} .
$$

После непосредственного вычисления интегралов это неравенство приводится к виду

$$
\left(e^{s}-1\right)^{2}<3\left(e^{s}-1\right)^{2}\left(s e^{s}-e^{s}+1\right)+4\left(s e^{s}-e^{s}+1\right) .
$$

Ясно, что полученное неравенство верно при $s>1$. Покажем, что при $s \leqslant 1$ верно неравенство

$$
\varphi(s)=-\left(e^{s}-1\right)^{2}+4\left(s e^{s}-e^{s}+1\right)>0 .
$$

Заметим, что $\varphi(0)=0$ и

$$
\varphi^{\prime}(s)=-2\left(e^{s}-1\right) e^{s}+4 s e^{s}=2 e^{s}\left(2 s+1-e^{s}\right)>0
$$

на отрезке $[0,1]$, что доказывает нужное соотношение. Таким образом,

$$
F(r, s) \leqslant 4 F(0, s)
$$

Докажем теперь оценку $4^{-1} F(0, s) \leqslant F(r, s)$ :

$$
F(r, s)=\frac{P(r, 0)+P(0, s)}{(Q(r, 0)+Q(0, s))^{2}} \geqslant 2^{-1} \frac{P(r, 0)+P(0, s)}{Q(r, 0)^{2}+Q(0, s)^{2}} .
$$


Поскольку

$$
F(r, 0)=\frac{P(r, 0)}{Q(r, 0)^{2}} \geqslant \frac{1}{2}
$$

то

$$
2^{-1} \frac{P(r, 0)+P(0, s)}{Q(r, 0)^{2}+Q(0, s)^{2}} \geqslant 4^{-1} \frac{(Q(r, 0))^{2}+P(0, s)}{Q(r, 0)^{2}+Q(0, s)^{2}} .
$$

В силу возрастания функции $\frac{t+a}{t+b}$ при $t>0,0<a<b$, а также с учетом неравенства $F(0, s)<1$ из следствия 2.2 , последнее выражение не меньше, чем

$$
4^{-1} \frac{P(0, s)}{Q(0, s)^{2}}=4^{-1} F(0, s),
$$

что доказывает требуемое.

Теперь докажем оценку $F(r, s) \leqslant 4 F(r, 0)$. Имеем

$$
F(r, s)=\frac{P(r, 0)+P(0, s)}{(Q(r, 0)+Q(0, s))^{2}} \leqslant \frac{P(r, 0)+P(0, s)}{Q(r, 0)^{2}+Q(0, s)^{2}} \leqslant 1,
$$

так как $F(r, 0) \leqslant 1$ и $F(0, s) \leqslant 1$ и верно неравенство $\frac{a_{1}+a_{2}}{b_{1}+b_{2}} \leqslant 1$, если $\frac{a_{1}}{b_{1}} \leqslant 1$ и $\frac{a_{2}}{b_{2}} \leqslant 1$. В то же время $F(r, 0) \geqslant \frac{1}{2}$, значит, $F(r, s) \leqslant 2 F(r, 0) \leqslant 4 F(r, 0)$.

Лемма 2.3. Пусть $a \leqslant r \leqslant s \leqslant b$, тогда $F(a, b) \leqslant 16 F(r, s)$.

ДокАзАтельство. Рассмотрим пять случаев, доказательства которых непосредственно вытекают из леммы 2.1, следствия 2.2 и леммы 2.2 .

1. Пусть $0 \leqslant a$. Тогда $F(a, b) \leqslant F(a, s) \leqslant F(r, s)$.

2. Пусть $b \leqslant 0$. Тогда согласно п. 1 леммы 2.1 имеем $F(a, b) \leqslant F(a, s)$. Произведем замену переменной:

$$
\begin{aligned}
F(a, s) & =\int_{a}^{s}|t| e^{t} d t\left(\int_{a}^{s} e^{t} d t\right)^{-2}=e^{-s} \int_{a-s}^{0}|t+s| e^{t} d t\left(\int_{a-s}^{0} e^{t} d t\right)^{-2} \\
& =e^{-s} \int_{a-s}^{0}|t| e^{t} d t\left(\int_{a-s}^{0} e^{t} d t\right)^{-2}+e^{-s}|s| \int_{a-s}^{0} e^{t} d t\left(\int_{a-s}^{0} e^{t} d t\right)^{-2} .
\end{aligned}
$$

Теперь воспользуемся тем, что $F(a-s, 0) \leqslant 1$, а также тем, что $[r-s, 0] \subset$ $[a-s, 0]$. Тогда последнее выражение оценится через

$$
\begin{aligned}
e^{-s} & +e^{-s}|s| \int_{r-s}^{0} e^{t} d t\left(\int_{r-s}^{0} e^{t} d t\right)^{-2} \\
& \leqslant 2 e^{-s} F(r-s, 0)+2 e^{-s} \int_{r-s}^{0}|s| e^{t} d t\left(\int_{r-s}^{0} e^{t} d t\right)^{-2}=2 F(r, s),
\end{aligned}
$$

где в последнем неравенстве использована оценка $F(r-s, 0) \geqslant 2^{-1}$.

3. Пусть $r \leqslant 0 \leqslant s$. Тогда $F(a, b) \leqslant 4 F(0, b) \leqslant 4 F(0, s) \leqslant 16 F(r, s)$.

4. Пусть $a \leqslant 0 \leqslant r$. Тогда $F(a, b) \leqslant 4 F(0, b) \leqslant 4 F(0, s) \leqslant 4 F(r, s)$.

5. Пусть $s \leqslant 0 \leqslant b$. Тогда $F(a, b) \leqslant 4 F(a, 0) \leqslant 4 \leqslant 8 F(r, 0) \leqslant 8 F(r, s)$. 
ЗАмЕЧАНИЕ 2.1. Пусть $\mu$ - выпуклая мера и $g$ - положительно однородная выпуклая функция, т.е. $g$ выпукла и $g(\lambda x)=|\lambda| g(x)$. Тогда если $\mu\left(g^{-1}(\{1\})\right)>0$, то $\mu\left(g^{-1}([0,1))\right)=0$.

Обоснование нетрудно усмотреть из логарифмической вогнутости меры. Действительно, положим $V=g^{-1}([0,1])$. Тогда $V$ - выпуклое множество, причем $\mu(V)>0$ и для некоторого $\delta>0$ верно неравенство $\mu(\alpha V) \leqslant \mu(V)-\delta$ для всякого положительного $\alpha<1$. Пусть найдется такое $0<c<1$, что $\mu(c V)>0$. Тогда, пользуясь неравенством выпуклости для меры и учитывая выпуклость $V$, имеем

$$
\mu(V)^{\alpha} \mu(c V)^{1-\alpha} \leqslant \mu(\alpha V+(1-\alpha)(c V))=\mu((c+\alpha(1-c)) V)<\mu(V)-\delta,
$$

что приводит к противоречию при $\alpha \rightarrow 1$.

Лемма 2.4. Пусть $\mu$ - выпуклая радоновская мера на локально выпуклом пространстве Е и $U$ - выпуклое открытое множество положительной меры. Тогда $\mu(U)=\mu(\bar{U})$, где $\bar{U}-$ замыкание множества $U$.

ДокАЗАТЕЛЬСтво. Без ограничения общности считаем, что $0 \in U$. Пусть $\widetilde{U}=\bigcap_{t>1} t U$. Покажем, что $\widetilde{U}=\bar{U}$. Легко видеть, что $\widetilde{U} \subset \bar{U}$. Проверим обратное включение. Пусть $x \notin \widetilde{U}$, значит, найдется такое $t>1$, что $x \notin t U$. По теореме Хана-Банаха найдется такой непрерывный линейный функционал $\ell$, что $\sup \{\ell(y), y \in t U\} \leqslant \ell(x)$. Поэтому

$$
\sup \{\ell(y), y \in U\} \leqslant t^{-1} \ell(x)<\ell(x),
$$

значит, $x \notin \bar{U}$. Теперь можно воспользоваться замечанием 2.1 для функционала Минковского множества $U$, либо непосредственно использовать выпуклость меры:

$$
\mu(U)^{2}=\mu\left(\frac{1}{2}(2-t) U+\frac{1}{2} t U\right)^{2} \geqslant \mu((2-t) U) \mu(t U) .
$$

Полагая $t \rightarrow 1$ в этом неравенстве, в пределе получаем $\mu(U) \geqslant \mu(\bar{U})$.

Отметим, что лемму 2.4 можно было вывести из замечания 2.1.

Теперь мы готовы приступить к доказательству теоремы 2.1.

ДокАЗАТЕЛЬСтво ТЕОРЕмЫ 2.1. Аналогично теореме 1.1 (только многочлен нужно продолжать по непрерывности) случай неполного пространства $E$ сводится к случаю полного, поэтому считаем пространство $E$ полным.

Несложно видеть, что достаточно доказать оценку только для непрерывных многочленов степени $d$. Сначала рассмотрим случай, когда $U$ является конечным объединением открытых выпуклых множеств положительной меры. По лемме 2.4 имеем $\mu(U)=\mu(\bar{U})$. Легко заметить, что $I_{U}$ - индикатор множества $U$ - является полунепрерывной снизу функцией, а $|f| I_{\bar{U}}-$ полунепрерывная сверху функция. Рассмотрим функции $f_{1}=I_{U}, f_{2}=|f|, f_{3}=1$ и $f_{4}=(C d)^{2 d}|f| I_{\bar{U}}$. Тогда интересующее нас неравенство

$$
(\mu(U))^{d+1} \int_{E}|f| d \mu \leqslant(C d)^{2 d} \int_{U}|f| d \mu
$$


обратится в соотношение

$$
\left(\int_{E} f_{1} d \mu\right)^{d+1} \int_{E} f_{2} d \mu \leqslant\left(\int_{E} f_{3} d \mu\right)^{d+1} \int_{E} f_{4} d \mu .
$$

Следствие 2.1 позволяет нам проверять это соотношение только для всех отрезков $\Delta \subset E$ и для всех мер $\nu$ с плотностями вида $\exp \ell$, где $\ell$ - аффинная функция на $\Delta$. Так как прямая пересекает конечное объединение открытых выпуклых множеств по конечному объединению интервалов и $\overline{\Delta \cap U} \subset \Delta \cap \bar{U}$, а ограничение многочлена на прямую - многочлен, то наша задача свелась к проверке для всякого одномерного многочлена $f$ следующего неравенства:

$$
\left(\int_{J} e^{k t+m} d t\right)^{d+1} \int_{a}^{b}|f(t)| e^{k t+m} d t \leqslant(C d)^{2 d} \int_{J}|f(t)| e^{k t+m} d t\left(\int_{a}^{b} e^{k t+m} d t\right)^{d+1}
$$

где $J$ является конечным объединением отрезков: $J=\bigcup_{k=1}^{n} J_{k}, J_{k}=\left[r_{k}, s_{k}\right]$. С помощью линейной замены задача сводится к проверке для всякого многочлена $f$ соотношения

$$
\left(\int_{J} e^{t} d t\right)^{d+1} \int_{a}^{b}|f(t)| e^{t} d t \leqslant(C d)^{2 d} \int_{J}|f(t)| e^{t} d t\left(\int_{a}^{b} e^{t} d t\right)^{d+1}
$$

Далее сведем случай произвольного объединения отрезков к случаю одного отрезка, а в случае одного отрезка оценку будем сводить к оценке для многочлена первой степени.

Пусть сначала $J=[r, s]$. Напомним, что

$$
Q(r, s)=\int_{r}^{s} e^{t} d t
$$

Докажем следующую оценку:

$$
Q(r, s)^{d+1} \int_{a}^{b}|f(t)| e^{t} d t \leqslant\left(C_{0} d\right)^{d} \int_{r}^{s}|f(t)| e^{t} d t Q(a, b)^{d+1} .
$$

По теореме 1.4 верна оценка $\|f\|_{1} \leqslant\left(c_{1} d\right)^{d}\|f\|_{0}$, где $c_{1}$ - универсальная константа. Пусть $f(t)=A \prod\left(t-t_{k}\right)$. Тогда, применяя теорему $1.4 \mathrm{k}$ вероятностной мере $(Q(a, b))^{-1} e^{t} d t$, получаем

$$
\begin{aligned}
& Q(r, s)^{d+1} \int_{a}^{b}|f(t)| e^{t} d t \\
& \leqslant\left(c_{1} d\right)^{d} A Q(r, s)^{d+1} Q(a, b) \exp \left(Q(a, b)^{-1} \int_{a}^{b} \ln \left(\prod_{k=1}^{d}\left|t-t_{k}\right|\right) e^{t} d t\right) \\
& \quad=\left(c_{1} d\right)^{d} A Q(r, s)^{d+1} Q(a, b) \prod_{k=1}^{d} \exp \left(Q(a, b)^{-1} \int_{a}^{b} \ln \left(\left|t-t_{k}\right|\right) e^{t} d t\right)
\end{aligned}
$$


Пользуясь тем, что $\left\|t-t_{k}\right\|_{0} \leqslant\left\|t-t_{k}\right\|_{1}$, последнее можно оценить выражением

$$
\begin{array}{rl}
\left(c_{1} d\right)^{d} & A Q(a, b)^{1-d} Q(r, s)^{d+1} \prod_{k=1}^{d} \int_{a}^{b}\left|t-t_{k}\right| e^{t} d t \\
& =\left(c_{1} d\right)^{d} A Q(a, b)^{1-d} Q(r, s)^{1-d} \prod_{k=1}^{d} Q(r, s)^{2} \int_{a}^{b}\left|t-t_{k}\right| e^{t} d t .
\end{array}
$$

Положим $t_{k}=\alpha_{k}+i \beta_{k}$. По лемме 2.3 имеем

$$
\begin{aligned}
& Q(r, s)^{2} \int_{a}^{b}\left|t-t_{k}\right| e^{t} d t \leqslant Q(r, s)^{2} \int_{a}^{b}\left|t-\alpha_{k}\right| e^{t} d t+Q(r, s)^{2} \int_{a}^{b}\left|\beta_{k}\right| e^{t} d t \\
& \quad \leqslant 16 Q(a, b)^{2} \int_{r}^{s}\left|t-\alpha_{k}\right| e^{t} d t+16 Q(a, b)^{2} \int_{r}^{s}\left|\beta_{k}\right| e^{t} d t \\
& \quad \leqslant 32 Q(a, b)^{2} \int_{r}^{s}\left|t-t_{k}\right| e^{t} d t .
\end{aligned}
$$

Таким образом,

$$
\begin{aligned}
& \left(c_{1} d\right)^{d} A Q(a, b)^{1-d} Q(r, s)^{1-d} \prod_{k=1}^{d} Q(r, s)^{2} \int_{a}^{b}\left|t-t_{k}\right| e^{t} d t \\
& \leqslant\left(c_{1} d\right)^{d} A Q(a, b)^{1-d} Q(r, s)^{1-d} \prod_{k=1}^{d} 32 Q(a, b)^{2} \int_{r}^{s}\left|t-t_{k}\right| e^{t} d t .
\end{aligned}
$$

Вновь воспользовавшись оценкой из теоремы 1.4, получим следующую оценку сверху для правой части:

$$
\begin{aligned}
& \left(32 c_{1} d\right)^{d} A Q(a, b)^{d+1} Q(r, s) \prod_{k=1}^{d} c_{1} \exp \left(Q(r, s)^{-1} \int_{r}^{s} \ln \left|t-t_{k}\right| e^{t} d t\right) \\
& =\left(32 c_{1}^{2} d\right)^{d} A Q(a, b)^{d+1} Q(r, s) \exp \left(Q(r, s)^{-1} \int_{r}^{s} \ln \prod_{k=1}^{d}\left|t-t_{k}\right| e^{t} d t\right) \\
& \leqslant\left(32 c_{1}^{2} d\right)^{d} Q(a, b)^{d+1} \int_{r}^{s}|f(t)| e^{t} d t .
\end{aligned}
$$

Последняя оценка использует неравенство $\|f\|_{0} \leqslant\|f\|_{1}$. Таким образом, случай, когда $J$ - один отрезок, разобран.

Пусть теперь $J=\bigcup_{k=1}^{n} J_{k}$, где $J_{k}=\left[r_{k}, s_{k}\right]$ - невырожденные отрезки. Рассмотрим функцию

$$
G\left(r_{2}, s_{2}, \ldots, r_{n-1}, s_{n-1}\right)=\sum_{k=1}^{n} \int_{r_{k}}^{s_{k}}|f(t)| e^{t} d t\left(\sum_{k=1}^{n} \int_{r_{k}}^{s_{k}} e^{t} d t\right)^{-d-1} .
$$

Пусть $n \geqslant d+4$. Исследуем функцию $G$ на минимум на компактном множестве $s_{1} \leqslant r_{2} \leqslant \cdots \leqslant s_{n-1} \leqslant r_{n}$ (считаем $r_{1}, s_{1}, r_{n}$ и $s_{n}$ фиксированными). Так как функция $G$ гладкая на данном компакте, то минимум существует и достигается либо внутри области, либо на ее границе. Предположим, 
что минимум этой функции достигается внутри области, т.е. на множестве $s_{1}<r_{2}<\cdots<s_{n-1}<r_{n}$. Тогда в точке минимума все частные производные этой функции обратятся в нуль:

$$
\begin{aligned}
0 & =\partial_{s_{j}} G\left(r_{2}, s_{2}, \ldots, r_{n-1}, s_{n-1}\right) \\
& =\left(e^{s_{j}}\left|f\left(s_{j}\right)\right| \sum_{k=1}^{n} \int_{r_{k}}^{s_{k}} e^{t} d t-(d+1) e^{s_{j}} \sum_{k=1}^{n} \int_{r_{k}}^{s_{k}}|f(t)| e^{t} d t\right)\left(\sum_{k=1}^{n} \int_{r_{k}}^{s_{k}} e^{t} d t\right)^{-d-2}, \\
0 & =\partial_{r_{j}} G\left(r_{2}, s_{2}, \ldots, r_{n-1}, s_{n-1}\right) \\
& =\left(-e^{r_{j}}\left|f\left(r_{j}\right)\right| \sum_{k=1}^{n} \int_{r_{k}}^{s_{k}} e^{t} d t+(d+1) e^{r_{j}} \sum_{k=1}^{n} \int_{r_{k}}^{s_{k}}|f(t)| e^{t} d t\right)\left(\sum_{k=1}^{n} \int_{r_{k}}^{s_{k}} e^{t} d t\right)^{-d-2} .
\end{aligned}
$$

Следовательно, для всяких $j, k$ из множества $\{2,3, \ldots, n-1\}$ имеем

$$
\left|f\left(r_{k}\right)\right|=\left|f\left(r_{j}\right)\right|=\left|f\left(s_{k}\right)\right|=\left|f\left(s_{j}\right)\right|,
$$

что невозможно для многочлена степени $d$, ибо $2(n-2) \geqslant 2 d+2$. Таким образом, минимум $G$ достигается на границе, значит, минимум отношения

$$
\sum_{k=1}^{n} \int_{r_{k}}^{s_{k}}|f(t)| e^{t} d t\left(\sum_{k=1}^{n} \int_{r_{k}}^{s_{k}} e^{t} d t\right)^{-d-1}
$$

по всевозможным конечным объединениям отрезков достигается на наборе, в котором число отрезков не превосходит $d+4$. Поэтому в доказательстве общего случая можно считать, что $n$ - количество отрезков в $J$ - не больше $d+4$.

Выведем общий случай из доказанного для отрезка:

$$
\begin{aligned}
& \left(\sum_{k=1}^{n} Q\left(r_{k}, s_{k}\right)\right)^{d+1} \int_{a}^{b}|f(t)| e^{t} d t \leqslant n^{d} \sum_{k=1}^{n} Q\left(r_{k}, s_{k}\right)^{d+1} \int_{a}^{b}|f(t)| e^{t} d t \\
& \leqslant n^{d} \sum_{k=1}^{n}\left(32 c_{1}^{2} d\right)^{d} \int_{r_{k}}^{s_{k}}|f(t)| e^{t} d t Q(a, b)^{d+1} \\
& \leqslant(C d)^{2 d} \sum_{k=1}^{n} \int_{r_{k}}^{s_{k}}|f(t)| e^{t} d t Q(a, b)^{d+1} .
\end{aligned}
$$

Случай, когда $U$ - произвольное измеримое множество, несложно доказывается предельным переходом. Действительно, в силу радоновости меры, внутри $U$ есть компакт сколь угодно близкой меры. Компакт, в свою очередь, можно покрыть открытым множеством близкой меры, которое является объединением выпуклых открытых множеств из базы топологии. Значит, компакт лежит в конечном объединении выпуклых открытых множеств. Поэтому $U$ приближается по симметрической разности конечными объединениями открытых выпуклых множеств, для которых оценка уже доказана. Теорема 2.1 доказана.

Отметим, что вместо теоремы 1.4 в данном доказательстве можно было использовать аналогичный результат С. Г. Бобкова, но для непрерывных многочленов в конечномерном пространстве (см. [4]), а на некоторых этапах доказательства - даже результат работы [7] об оценке $\|g\|_{1}$ через $\|g\|_{0}$ для полунорм $g$. 
ЗАмЕчАНИЕ 2.2. Из доказательства видно, что если $U$ - объединение $n$ открытых выпуклых множеств, то справедлива оценка

$$
\mu(U)^{d+1} \int_{E}|f| d \mu \leqslant(C n d)^{d} \int_{U}|f| d \mu
$$

где $C$ - некоторая абсолютная постоянная.

Обозначим через $\mathscr{P}_{\text {fin }}^{d}(\mu)$ совокупность всех измеримых полиномов, которые являются пределами по мере последовательностей многочленов вида $f\left(\ell_{1}\right.$, $\left.\ldots, \ell_{n}\right)$, где $\ell_{i} \in E^{*}$ и $f$ - многочлен на $\mathbb{R}^{n}$ степени $d$. Это множество совпадает с замыканием в $L^{1}(\mu)$ многочленов того же типа, что легко видеть, например, из следствия 1.2. Заметим, что оценку из теоремы 2.1 для $f \in \mathscr{P}_{\text {fin }}^{d}(\mu)$ можно было бы получить, используя только конечномерную локализационную лемму, приближая произвольные множества цилиндрами. Именно для полиномов из $\mathscr{P}_{\text {fin }}^{d}(\mu)$ В. Е. Бережным в статье [5] и был обобщен результат, приведенный в статье С. Г. Бобкова [4].

Отметим, что для общих мер

$$
\mathscr{P}_{\text {fin }}^{d}(\mu) \subset \mathscr{P}_{\text {cont }}^{d}(\mu) \subset \mathscr{P}_{\text {mes }}^{d}(\mu)
$$

однако для гауссовских мер все три класса совпадают. Для пространств с топологическими базисами имеет место равенство

$$
\mathscr{P}_{\text {fin }}^{d}(\mu)=\mathscr{P}_{\text {cont }}^{d}(\mu) \text {. }
$$

Для общего случая выпуклой меры даже в простейших пространствах типа $l^{2}$ или $\mathbb{R}^{\infty}$ вопрос о соотношении этих классов с $\mathscr{P}_{\text {mes }}^{d}(\mu)$ остается открытым.

Из теоремы 2.1 и следствия 1.2 вытекает следующий полезный факт.

СледСтвие 2.3. Пусть последовательность многочленов из $\mathscr{P}_{\mathrm{cont}}^{d}$ cходится по мере на некотором множестве положительной меры. Тогда эта последовательность сходится во всех $L^{p}$ на всем пространстве.

Отметим еще, что для мер, являющихся сужениями выпуклых мер на подмножества положительной меры, все $L^{p}$-нормы на пространстве непрерывных многочленов эквивалентны.

Из теоремы 2.1 можно независимо вывести закон нуля или единицы для линий уровня непрерывного многочлена.

СледСтвие 2.4. Пусть $f \in \mathscr{P}_{\text {cont }}^{d}(\mu) u U_{c}(f)=\{x \in E: f(x)=c\}$. Тогда величина $\mu\left(U_{c}(f)\right)$ равна либо 0, либо 1 .

ДокАЗАТЕЛЬСтво. Пусть $\mu\left(U_{c}(f)\right)>0$, тогда по теореме 2.1 имеем

$$
\mu\left(U_{c}(f)\right)^{d+1} \int_{E}|f-c| d \mu \leqslant(C d)^{2 d} \int_{U_{c}(f)}|f-c| d \mu=0 .
$$

Таким образом, $\mu$-п.в. выполняется равенство $f=c$. 


\section{Список литературы}

[1] В. И. Богачев, Гауссовские меры, Наука, М., 1997, 352 с.; англ. пер.: V. I. Воgachev, Gaussian measures, Math. Surveys Monogr., 62, Amer. Math. Soc., Providence, RI, 1998, xii+433 pp.

[2] V. I. Bogachev, "Gaussian measures on infinite-dimensional spaces", Real and stochastic analysis, World Sci. Publ., Hackensack, NJ, 2014, 1-83.

[3] C. Borell, "Convex measures on locally convex spaces", Ark. Mat., 12:1-2 (1974), 239-252.

[4] S. G. Bobkov, "Remarks on the growth of $L^{p}$-norms of polynomials", Geometric aspects of functional analysis, Lecture Notes in Math., 1745, Springer, Berlin, 2000, $27-35$.

[5] V. Berezhnoy, "On the equivalence of integral norms on the space of measurable polynomials with respect to a convex measure", Theory Stoch. Process., 14:1 (2008), $7-10$.

[6] J. Bourgain, "On the distribution of polynomials on high dimensional convex sets", Geometric aspects of functional analysis (1989-90), Lecture Notes in Math., 1469, Springer, Berlin, 1991, 127-137.

[7] R. Latała, "On the equivalence between geometric and arithmetic means for log-concave measures", Convex geometric analysis (Berkeley, CA, 1996), Math. Sci. Res. Inst. Publ., 34, Cambridge Univ. Press, Cambridge, 1999, 123-127.

[8] V. I. Bogachev, Measure theory, v. 2, Springer-Verlag, Berlin, 2007, xiv+575 pp.

[9] V.I. Bogachev, Differentiable measures and the Malliavin calculus, Math. Surveys Monogr., 164, Amer. Math. Soc., Providence, RI, 2010, xvi+488 pp.

[10] Л. М. Арутюнян, И.С. Ярославцев, "Об измеримых многочленах на бесконечномерных пространствах", Докл. РАН, 449:6 (2013), 627-631; англ. пер.: L. M. Arutyunyan, I.S. Yaroslavtsev, "On measurable polynomials on infinitedimensional spaces", Dokl. Math., 87:2 (2013), 214-217.

[11] Л. М. Арутюнян, Е.Д. Косов, И. С. Ярославцев, "О некоторых свойствах многочленов, измеримых по гауссовской мере”, Докл. РАН, 457:2 (2014), 131-135; англ. пер.: L. M. Arutyunyan, E. D. Kosov, I. S. Yaroslavtsev, "On some properties of polynomials measurable with respect to a Gaussian measure", Dokl. Math., 90:1 (2014), 419-423.

[12] S. G. Bobkov, J. Melbourne, Hyperbolic measures on infinite dimensional spaces, arXiv: 1405.2961.

[13] Ф. Назаров, М. Содин, А. Вольберг, "Геометрическая лемма Каннана-ЛовасаШимоновича, не зависящие от размерности оценки распределения значений полиномов и распределение нулей случайных аналитических функций", Алгебра и анализ, 14:2 (2002), 214-234; англ. пер.: F. Nazarov, M. Sodin, A. Vol'berg, "The geometric Kannan-Lovász-Simonovits lemma, dimension-free estimates for the distribution of the values of polynomials, and the distribution of the zeros of random analytic functions", St. Petersburg Math. J., 14:2 (2003), 351-366.

[14] E. J. Remez, "Sur une propriété extremale des polynômes de Tschebychef", Сообщ. Харък. мат. о-ва, 13:1 (1936), 93-95.

[15] P. Borwein, T. Erdélyi, Polynomials and polynomial inequalities, Grad. Texts in Math., 161, Springer-Verlag, New York, 1995, x+480 pp.

[16] С. Г. Бобков, "Некоторые обобщения результатов Ю. В. Прохорова о неравенствах типа Хинчина для полиномов", ТВП, 45:4 (2000), 745-748; англ. пер.: S. G. Bobkov, "Some generalizations of Prokhorov's results on Khinchin-type inequalities for polynomials", Theory Probab. Appl., 45:4 (2001), 644-647.

[17] A. Carbery, J. Wright, "Distributional and $L^{q}$ norm inequalities for polynomials over convex bodies in $\mathbb{R}^{n "}$, Math. Res. Lett., 8:3 (2001), 233-248. 
[18] L. Lovász, M. Simonovits, "Random walks in a convex body and an improved volume algorithm", Random Structures Algorithms, 4:4 (1993), 359-412.

[19] R. Kannan, L. Lovász, M. Simonovits, "Isoperimetric problems for convex bodies and a localization lemma", Discrete Comput. Geom., 13:1 (1995), 541-559.

\section{Лаврентин Мартунович Арутюнян}

(Lavrentin M. Arutyunyan)

Механико-математический факультет

Московского государственного университета

им. М. В. Ломоносова

E-mail: Lavrentin@ya.ru

\section{Егор Дмитриевич Косов}

(Egor D. Kosov)

Механико-математический факультет

Московского государственного университета

им. М. В. Ломоносова

E-mail: ked_2006@mail.ru
Поступила в редакцию 30.10.2014 и 02.12.2014 